\title{
Comparison of Multi-Gene Testing Data Between Fresh and Formalin-Fixed Specimens From Core Needle Biopsy in Patients With NSCLC
}

\begin{abstract}
Zhi-Gang Yao ${ }^{1}$, Zhi-Gang Wei ${ }^{2}$, Xian-Kui Cheng ${ }^{1}$, Guang-Hui Huang ${ }^{3}$, Yuan-Yuan Zong ${ }^{1}$, Min Meng ${ }^{3}$, Jia-Mei $\mathrm{Li}^{1}$, Xiao-Ying Han ${ }^{3}$, Jia-Wen Xu ${ }^{1}$, Jiao Wang ${ }^{3}$, Hai-Yan Jing ${ }^{1}$, Wen-Hong $\mathrm{Li}^{3}$, Zhi-Xin $\mathrm{Cao}^{1}$, Yang $\mathrm{Ni}^{3}$, Xi-Chao Sun ${ }^{1 * t}$, Xia Yang ${ }^{3 * t}$ and Xin $\mathrm{Ye}^{2 * t}$

${ }^{1}$ Department of Pathology, Shandong Provincial Hospital Affiliated to Shandong First Medical University, Jinan, China, ${ }^{2}$ Department of Oncology, The First Affiliated Hospital of Shandong First Medical University \& Shandong Provincial Qianfoshan Hospital, Shandong Key Laboratory of Rheumatic Disease and Translational Medicine, Shandong Lung Cancer Institute, Jinan, China, ${ }^{3}$ Department of Oncology, Shandong Provincial Hospital Affiliated to Shandong First Medical University, Jinan, China
\end{abstract}

OPEN ACCESS

Edited by: József Tímár,

Semmelweis University, Hungary

*Correspondence:

Xin Ye

yexintaian2020@163.com

Xia Yang

yangxjinan@163.com

Xi-Chao Sun

sun339168@126.com

tThese authors have contributed equally to this work

Received: 26 June 2021 Accepted: 11 November 2021 Published: 13 December 2021

Citation:

Yao ZG, Wei ZG, Cheng XK, Huang $G H$, Zong $Y Y$, Meng $M, L i J M$, Han $X Y, X u J W$, Wang J, Jing $H Y$, $L i W H$, Cao $Z X, N i Y$, Sun $X C$, Yang $X$ and $Y e X(2021)$ Comparison of MultiGene Testing Data Between Fresh and Formalin-Fixed Specimens From Core Needle Biopsy in Patients With NSCLC.

Pathol. Oncol. Res. 27:1609931. doi: 10.3389/pore.2021.1609931
Purpose: Currently, formalin-fixed paraffin-embedded (FFPE) tissue specimens are the conventional material for gene testing for non-small cell lung cancer (NSCLC) patients. In our study, we aimed to develop a quick gene testing procedure using fresh core needle biopsy samples from NSCLC patients.

Methods: In total, 77 fresh NSCLC samples obtained from core needle biopsy were evaluated by frozen section examination. If the NSCLC diagnosis and adequate tumor cell counts were confirmed by histopathology, the fresh tissues were used to extract DNA and subsequent gene testing by ARMS-PCR. Meanwhile, the paired FFPE core needle biopsy samples from 30 NSCLC patients also underwent gene testing.

Results: In total, 77 fresh samples showed an EGFR mutation rate of $61.0 \%$, higher than the levels in the Asian. Following a comparison of gene testing results with fresh tissues and paired FFPE tissues from the 30 patients, no significant difference in the DNA concentration extracted from fresh tissues and FFPE tissues was found. However, DNA purity was significantly higher in fresh tissues than that in FFPE tissues. Gene testing detected the same gene mutations in $93.3 \%$ of cases in fresh tissues and paired FFPE tissues. The gene testing procedure using fresh biopsy samples greatly shortens the waiting time of patients.

Conclusion: The multi-gene mutation testing using fresh core needle biopsy samples from NSCLC patients is a reasonable, achievable, and quick approach. Fresh tissues may serve as a potential alternative to FFPE tissues for gene testing in NSCLC patients.

Keywords: pathology, EGFR, lung cancers, frozen section examination, fresh frozen tissue 


\section{INTRODUCTION}

Patients with non-small cell lung cancer (NSCLC) often harbor driver mutations in multiple oncogenes, including EGFR, RAS, ALK, ROS1, BRAF, HER2, RET, etc., [1]. Gene mutation testing for lung cancer is important for identification of potentially efficacious targeted therapies. In particular, when it comes to patients with advanced NSCLC (stage III and IV), they have lost the opportunity of surgical therapy. As a result, gene mutations analysis is critical to developing the individualized clinical treatment strategy for those patients [1]. Currently, formalinfixed paraffin-embedded (FFPE) tissue specimens are a conventional and valuable source of sample material for mutation analysis. FFPE tissues effectively preserve the cellular, architectural, and morphological details and allow easy storage at room temperature for extensive periods. Moreover, FFPE section examination will provide much quality-controlled data of samples, including a pathological diagnosis and tumor cell count. However, much effort has been made to verify whether fresh materials are suitable for molecular testing in recent years. It has been demonstrated that EGFR mutations can be detected using fresh samples obtained by CT-guided core needle biopsy [2]. Lai et al. also performed EGFR mutation analysis using surgically resected fresh specimens [3]. These screening procedures using fresh materials have proven to be achievable and cost-effective.

In this study, we evaluated the multi-gene mutation status in fresh tissues and paired FFPE tissues from core needle biopsy for target therapies of patients with NSCLC. Notably, a frozen section examination was involved in the quality control of fresh biopsy tissues, including the pathological diagnosis and tumor cell count. We reasoned that the comparable results in the two materials should validate the mutation analysis using fresh tissues from a core needle lung biopsy.

\section{MATERIALS AND METHODS}

\section{CT-Guided Percutaneous Needle Lung Biopsy}

As previously described [4], 77 patients were placed in prone, supine, or lateral positions, whichever provided access to the best puncture pathway. All procedures were performed under CT (Neusoft CT, Neusoft Group Co., Ltd, China) guidance. A 16G sleeve-core needle (PRECISA fine-core needle, H. S. Hospital Service S. P. A, Aprilia, Italy) was pierced through the proximal edge of the lesion (Figure 1A). The core was then pulled out, with an 18G biopsy needle pierced into the lesion through the $16 \mathrm{G}$ sleeve. Each tumor
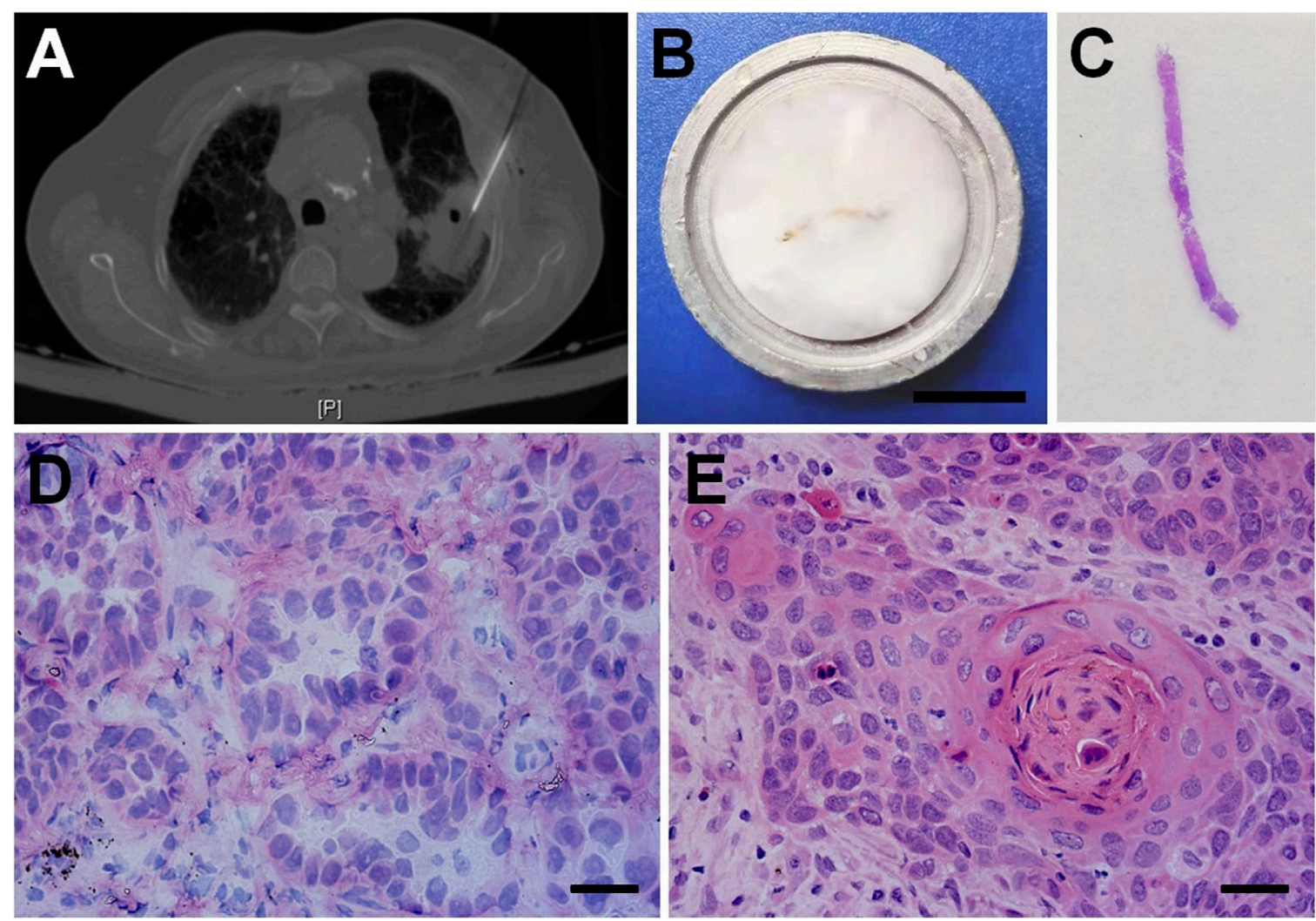

FIGURE 1 | CT-guided core needle lung biopsy and the frozen section diagnosis of NSCLC. (A) CT-guided percutaneous needle biopsy for pulmonary nodules. (B) The fresh biopsy sample was embedded in gel medium on a metal tissue disc and then frozen rapidly to about $-20^{\circ} \mathrm{C}$. Bar $=1 \mathrm{~cm}$. (C) The hemotoxylin-eosin (H\&E) staining for a frozen tissue section. Histopathologic features of frozen section in the rapid diagnosis of lung adenocarcinoma (D), squamous cell carcinoma (E). Bars $=100 \mu \mathrm{m}$. 
was sampled 2-3 strips of samples, each measuring 1-1.5 cm. After the biopsy, the obtained materials were sent to the Department of Pathology for pathology examination.

\section{Pathology Examination}

In the pathological laboratory, all experimental operations were carried out following standard biosecurity and institutional safety procedures. As shown in Figure 1B, the fresh biopsy samples were placed on a metal tissue disc and embedded in a gel medium (Kangleyi Inc. Jiangsu, China). The samples were then frozen rapidly to about $-20^{\circ} \mathrm{C}$ (we consider them fresh frozen tissues, the same below). The 3- $\mu \mathrm{m}$ thick tissues were cut and stained with hemotoxylin-eosin (H\&E) (Figure 1C). NSCLC was quickly diagnosed microscopically. For the patient with NSCLC, one strip of the entire fresh frozen (FF) tissues, which was rich in cancer cells (at least 200 cancer cells) examined by microscopy [5], was selected for DNA extraction. The rest of the strips were fixed in $10 \%$ formalin and prepared for paraffin sections and final pathology diagnosis. In our study, 30 patients with NSCLC confirmed by frozen section examination were enrolled to compare the results of multi-gene mutation testing using FF tissues and paired FFPE tissues.

\section{DNA Extraction}

For FF tissues, DNA was extracted using the DNA kit (AmoyDx, Xiamen, China) according to the manufacturer's instructions. For paired FFPE tissues, biopsy samples with at least 200 cancer cells were serially sliced on $8-10$ slides with 5 - $\mu \mathrm{m}$ thick and collected in specific tubes [5]. The tissue was deparaffinized and extracted DNA using a DNA FFPE tissues Kit (AmoyDx, Xiamen, China) according to the manufacturer's instructions. The DNA purity extracted from FF tissues and FFPE tissues was measured with a NanoDrop spectrophotometer (Thermo Fisher Scientific, Waltham, MA, United States), and the OD260/280 ratio approximately equivalent to 1.8 was considered to be an ideal value.

\section{Multi-Gene Mutation Testing}

According to the manufacturer's protocol, amplification refractory mutation system (ARMS)-PCR was performed using AmoyDx Multi-Gene Mutations Detection Kit (AmoyDx, Xiamen, China) and AmoyDx MET Mutation Detection Kit (AmoyDx, Xiamen, China) to detect genes of EGFR, KRAS, BRAF, NRAS, HER2, and PIK3CA mutations, EML4-ALK, ROS1 and RET fusions, and MET Exon 14 skipping mutation.

\section{Efficiency of Gene Testing Using Fresh Tissues}

In order to compare the efficiency of gene testing using FF and paired FFPE tissues, the time interval between physicians requesting and obtaining a test result was calculated.

\section{Statistical Analysis}

We used SPSS 21.0 for statistical analysis. The differences between FF and paired FFPE tissues groups were analyzed using paired Student's t-tests. Results were expressed as mean \pm SEM. $p<0.05$ was considered to be statistically significant.

\section{RESULTS}

\section{The Frozen Section Examination of NSCLC}

The percutaneous CT-guided core needle biopsy is widely accepted as an accurate and safe procedure for the characterization of solid lung nodules (Figure 1A). The fresh biopsy samples were firstly dedicated to frozen section examination (Figures 1B,C). Pathologists were able to correctly identify lung adenocarcinoma (AC, Figure 2D) and squamous cell carcinoma (SCC, Figure 2E) with typical histological characteristics.

\section{Clinicopathological Characteristics of Patients}

As shown in Table 1, frozen section examination confirmed the NSCLC diagnosis and adequate tumor cell count in the fresh tissues from 77 patients, which consisted of 39 males (50.6\%) and 38 females (49.4\%) with a median age of 65 years (range, $42-85$ years) of which 32 were smokers (41.6\%) vs. 45 nonsmokers (58.4\%). Frozen section examination revealed 70 (90.9\%) AC, 6 (7.8\%) SCC, and 1 (1.3\%) adenosquamous carcinoma (ASC), which is consistent with the final pathological diagnosis using FFPE tissues. CT scanners displayed the masses mainly located in the superior lobe of the right lung (44.2\%) and the superior lobe of the left lung $(24.7 \%)$ with an average length of $3.8 \mathrm{~cm}$ and an average width of $2.8 \mathrm{~cm}$. According to the eighth edition of the TNM Classification of lung cancer, most NSCLC patients were in stage IV (68.8\%) followed by stage III (20.8\%).

To evaluate the difference in mutation testing between FF tissues and FFPE tissues, 30 patients with NSCLC in stage IV were randomly selected in our study. As listed in Table 2, the 30 patients consisted of 13 (43.3\%) males and 17 (56.7\%) females with a median age of 62.5 years (range $43-74$ years). In total, 27 (90.0\%) AC and 3 (10.0\%) SCC were confirmed by frozen section examinations. The final pathological diagnosis in paraffin sections was all in accordance with the frozen section diagnosis. All patients had lost the opportunity of surgical treatment due to extrathoracic metastases.

\section{DNA Quality Assessment}

The concentration and purity of extracted DNA from FF tissues and FFPE tissues were evaluated before the ARMS-PCR assay. Results showed there was no significant difference in the DNA concentration extracted from FF tissues and FFPE tissues (23.86 \pm 3.10 vs. $24.07 \pm$ 3.27, $p>0.05$, Figure 2A). As for DNA OD260/280 ratio, results showed DNA purity was significantly higher in FF tissues than that in FFPE tissues $(1.91 \pm 0.04$ vs. $1.80 \pm 0.05, p<0.05$, Figure $2 B$ )

\section{Gene Mutation Testing}

Among the 77 patients with NSCLC, 15 (19.5\%) patients displayed the wild-type status of genes. In total, 47 (61.0\%) patients carried EGFR mutations, including 33.8\% EGFR L858R mutation and 28.6\% EGFR 19-del mutation (Table 1). In contrast, a subset of patients displayed KRAS (7.8\%), HER2 (5.2\%), BRAF (2.6\%), and ROS1 (2.6\%) mutations. In addition, 5 

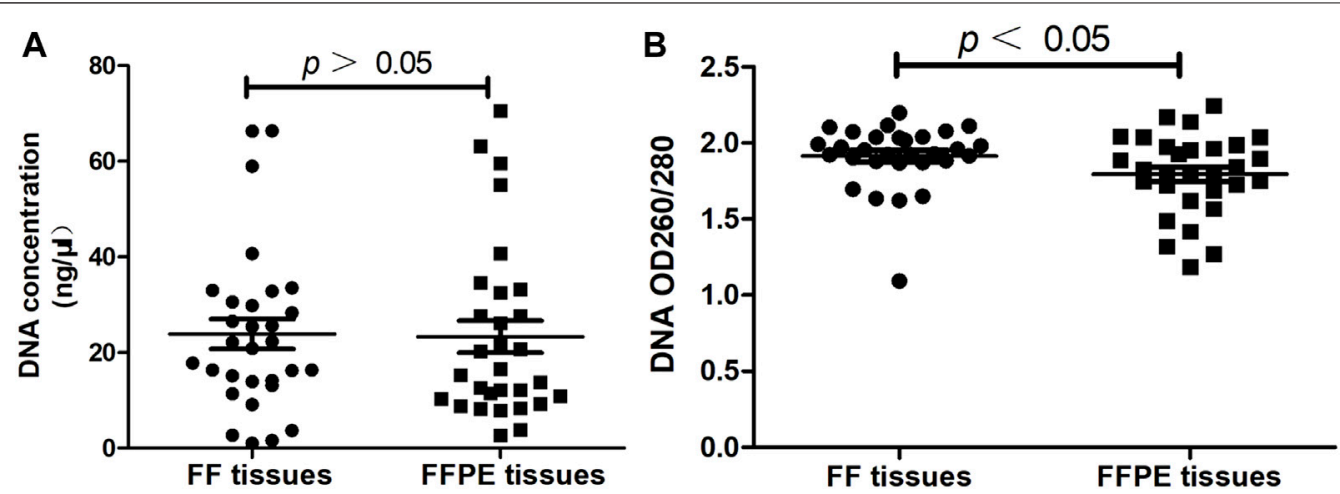

FIGURE 2 | Evaluating the quality of DNA extracted from FF tissues and paired FFPE tissues $(n=30)$. (A) The concentration of DNA. (B) The purity of DNA.

TABLE 1 | The clinicopathological characteristics of 77 patients.

\begin{tabular}{|c|c|c|}
\hline Characteristics & $\begin{array}{c}\text { No. of patients (total, } \\
77 \text { ) }\end{array}$ & Frequency (\%) \\
\hline \multicolumn{3}{|l|}{ Age (y) } \\
\hline$\geq 60$ & 51 & 66.2 \\
\hline$<60$ & 26 & 33.8 \\
\hline \multicolumn{3}{|l|}{ Gender } \\
\hline Male & 39 & 50.6 \\
\hline Female & 38 & 49.4 \\
\hline \multicolumn{3}{|l|}{ Smoking } \\
\hline Yes & 32 & 41.6 \\
\hline No & 45 & 58.4 \\
\hline \multicolumn{3}{|l|}{ Location } \\
\hline Right upper lobe & 34 & 44.2 \\
\hline Left upper lobe & 19 & 24.7 \\
\hline Right lower lobe & 13 & 16.9 \\
\hline Left lower lobe & 6 & 7.8 \\
\hline Right Middle lobe & 3 & 3.9 \\
\hline Left hilum & 2 & 2.6 \\
\hline \multicolumn{3}{|l|}{ TNM stage } \\
\hline IV & 53 & 68.8 \\
\hline III & 16 & 20.8 \\
\hline$\|$ & 5 & 6.5 \\
\hline 1 & 3 & 3.9 \\
\hline \multicolumn{3}{|l|}{ Pathological diagnosis } \\
\hline $\mathrm{AC}$ & 70 & 90.9 \\
\hline SCC & 6 & 7.8 \\
\hline ASC & 1 & 1.3 \\
\hline
\end{tabular}

Abbreviations: AC, adenocarcinoma; ASC, adenosquamous carcinoma; SCC, squamous cell carcinoma.

(6.5\%) patients harbored concomitant mutations in one or two of these genes.

Gene mutation testing was evaluated in 30 patients using FF tissues and paired FFPE tissues. We compared the cycle threshold (Ct) value of the PCR reaction between the systems using FF tissues and paired FFPE tissues. Results showed no significant difference in $\mathrm{Ct}$ value between the reactive systems using FF tissues and paired FFPE tissues $(24.43 \pm 0.51$ vs. $24.83 \pm 0.56, p>$ 0.05 , Figure 3$)$. We detected the same gene mutations in 28 (93.3\%) cases of FF tissues and paired FFPE tissues, including 13
(43.3\%) cases of EGFR L858R, 9 (30.0\%) cases of EGFR 19-del, 1 (3.3\%) case of HER-2 mutation, 1 (3.3\%) case of KRAS mutation, $1(3.3 \%)$ case of NRAS mutation, and $3(10.0 \%)$ cases of wild type. However, FF tissues in two cases of AC showed two gene mutations (Case 1: HER2, Figure 4A and EGFR L858R; Case 7: EGFR L858R, Figure 4C, and 19-del), while the paired FFPE tissues just displayed one gene mutation (Case 1: EGFR L858R, Figure 4B; Case 7: EGFR 19-del, Figure 4D). Histological examination showed a pure acinar pattern in the FF section and paired FFPE section (Figures 4A-D). Besides, FF tissues in two $(13.3 \%)$ cases showed fully inconsistent gene variants compared with paired FFPE tissues. In detail, FF tissues showed wild-type status (Case 19: Figure 4E) in one case of AC, while the paired FFPE tissues showed EGFR 20-ins (Case 19: Figure 4F). Histological analysis showed a pure acinar pattern in the FF section (Figure 4E), while a predominant micropapillary pattern (Figure 4F) was observed in the FFPE section. In another case of SCC, FF tissues showed PIK3CA mutation (Case 24: Figure 4G), while the paired FFPE tissues showed wild-type status (Case 24: Figure 4H). However, no histological difference was observed in the FF section and paired FFPE section (Figures 4G,H).

\section{Efficiency of the Gene Testing Using Fresh Tissues}

In order to analyze the efficiency of gene testing, we measured the time interval between physicians requesting and obtaining a test result. Results showed gene mutations testing using FF tissues had a significantly shorter time interval (1.73 \pm 0.12 days $)$ as compared to that using FFPE tissues $(3.60 \pm 0.15$ days, $p<$ 0.001, Figure 5).

\section{DISCUSSION}

Although lung cancer is the second most commonly occurring cancer worldwide (11.4\% of the newly diagnosed cancer cases), lung cancer is still the leading cause of cancer death $(18.0 \%$ of total cancer deaths) according to the latest data released by the International Agency for Research on Cancer (IARC) [6]. In 
TABLE 2 | The gene mutation results of 30 enrolled patients.

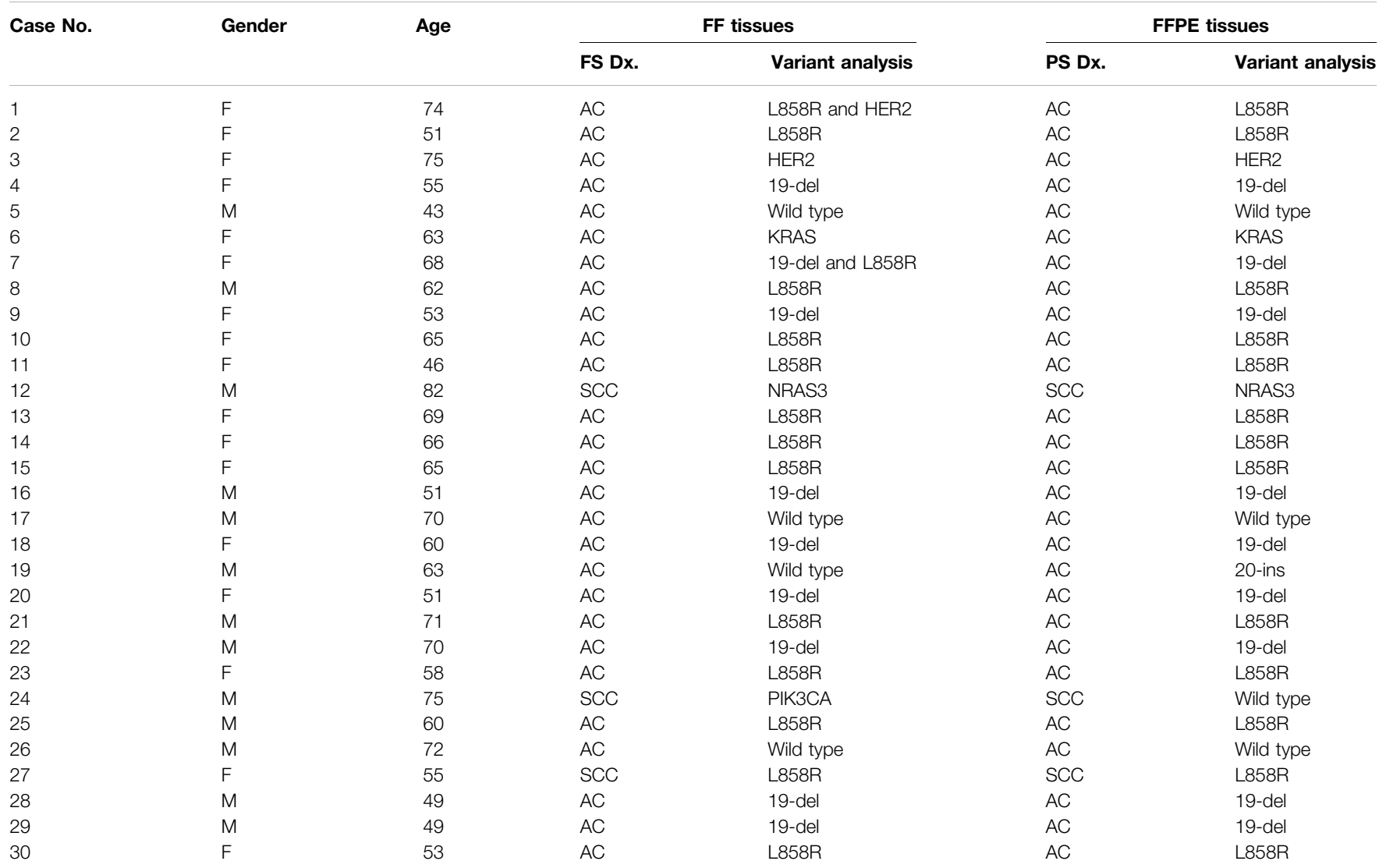

Abbreviations: AC, adenocarcinoma; Dx., diagnosis; F, female; FF, fresh frozen; FFPE, formalin-fixed paraffin-embedded; FS, frozen section; M, male; No., number; PS, paraffin section; SCC, squamous cell carcinoma.

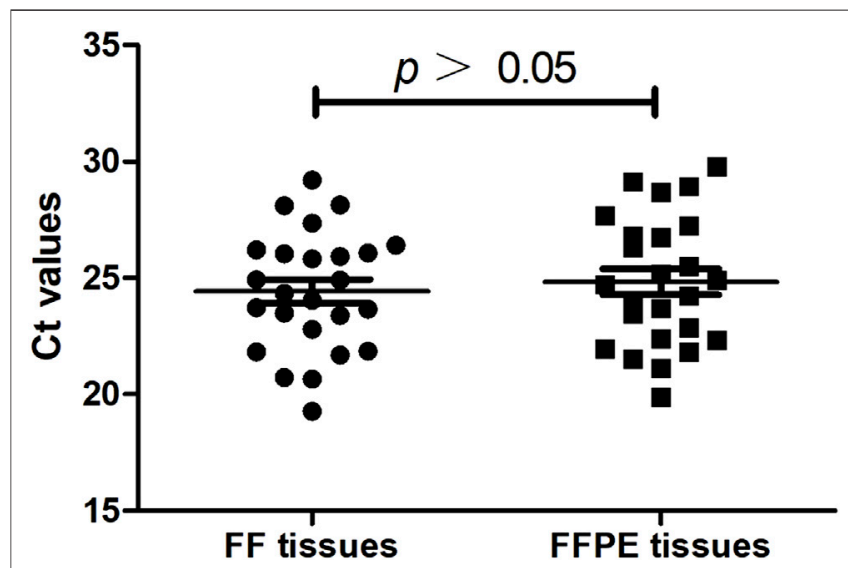

FIGURE 3 | The cycle threshold (Ct) value of the PCR reaction between the systems using FF tissues and paired FFPE tissues $(n=30)$.

China, lung cancer is the most commonly diagnosed cancer and the leading cause of cancer death. In 2014, the age-standardized incidence rate of lung cancer was 36.71 per 100,000 , while the age-standardized mortality rate was 28.49 per 100, 000 [7].
NSCLC comprises $85 \%$ of lung cancer and the majority of these patients are at an advanced stage when diagnosed [8]. For a subgroup of advanced NSCLC patients with gene mutations, they could benefit from corresponding targeted therapies, presenting as tumor shrink, acceptable side-effect profile, and improved overall survival [9]. Therefore, the relevant consensus and guidelines in China have emphasized the necessity and importance of assessment on driver gene status, the standardization of experimental operations, the acquisition and processing of samples, and the selection of methodologies and scenarios of implementation [10].

Normally, the standard techniques of gene mutation testing in advanced NSCLC are based on polymerase chain reaction (PCR) and DNA sequencing using needle biopsy FFPE samples. The advantages of FFPE tissue samples include technical ease and low storage cost come at the expense of the sample quality. However, there are some disadvantages in DNA molecular analysis using FFPE tissues, including additional deparaffinization step, DNA fragmentation, and poor quality, resulting from formaldehyde-driven crosslinking between proteins and nucleic acids [11]. Meanwhile, the main advantage of fresh material is that DNA is in optimal conditions, free from possible artifacts of the fixation and paraffin-embedded processes. This improves the efficiency of amplification, without the need for DNA purification [12]. 

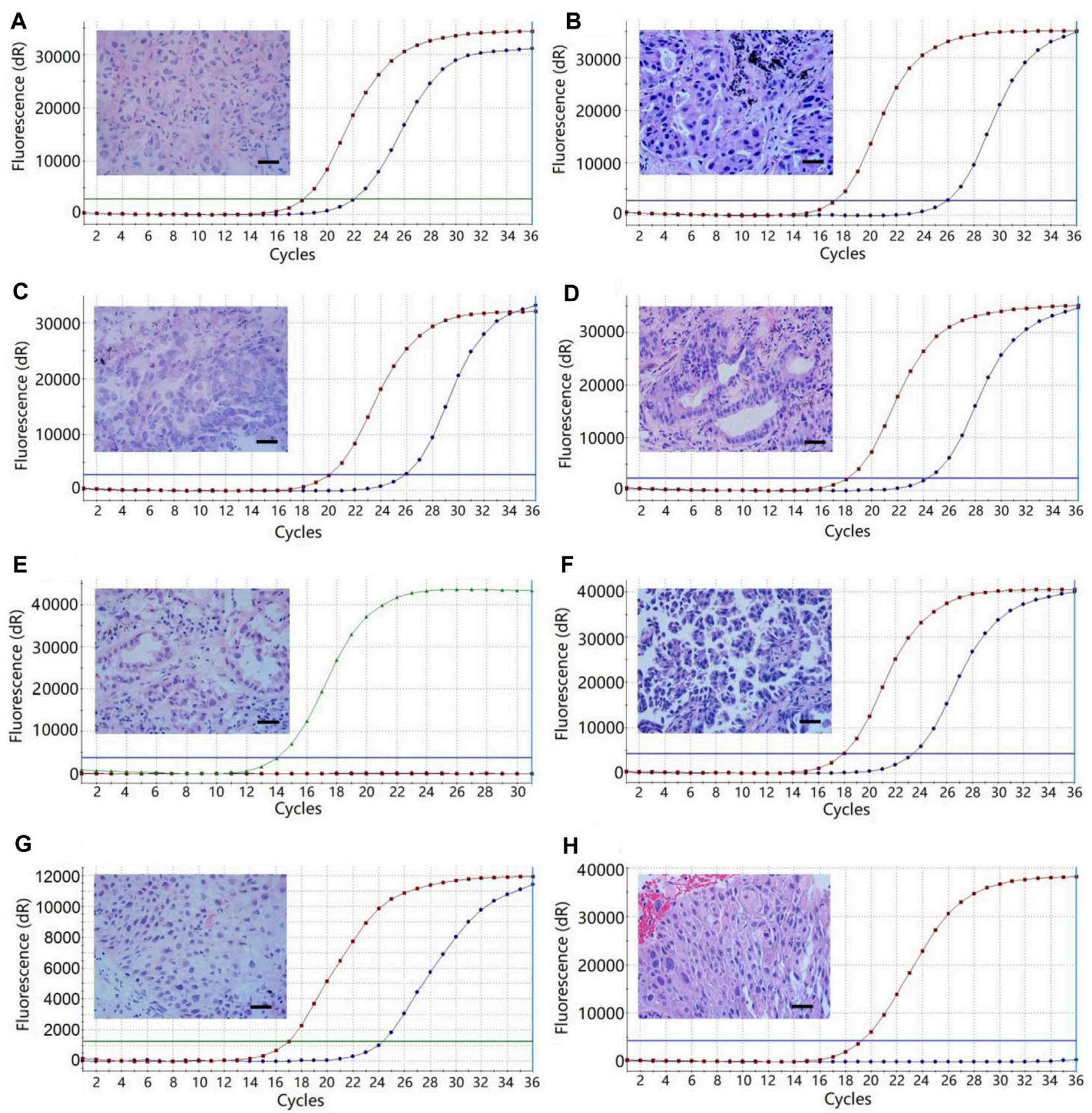

FIGURE 4 | The multi-gene mutation results of 30 patients using FF tissues and paired FFPE tissues. (A) Case 1, HER2 mutation of the lung adenocarcinoma with a predominant acinar pattern in FF tissue. (B) Case 1, EGFR L858R mutation of the lung adenocarcinoma with an acinar pattern in paired FFPE tissue. (C) Case 7, EGFR L858R mutation of the lung adenocarcinoma with a predominant acinar pattern in FF tissue. (D), Case 7, EGFR 19-del mutation of the lung adenocarcinoma with an acinar pattern in paired FFPE tissue. (E) Case 19, wild-type status of the lung adenocarcinoma with a predominant acinar pattern in FF tissue. (F) Case 19, EGFR 20-ins of the lung adenocarcinoma with a predominant micropapillary pattern. (G) Case 24, PIK3CA mutation of the squamous cell carcinoma in FF tissue. (H) Case 24, wild-type status of the squamous cell carcinoma in FFPE tissue. Bars $=100 \mu \mathrm{m}$. The red and green curves represent the positive controls, while the blue curves represent the tissue samples of NSCLC

Recently, many studies evaluated the quality of gene mutation testing using fresh and FFPE samples. Suciu et al. demonstrated the validity of EGFR gene mutations from fresh tissues in lung adenocarcinoma patients [13]. Spencer et al. [14] compared the variants of 27 cancerrelated genes between 16 pairs of FF and FFPE tissues from patients with lung carcinoma and found that the concordance rate was up to
$96.8 \%$ in the single-nucleotide variants. In our study, the gene mutation results showed high concordance $(93.3 \%)$ between FF tissues and paired FFPE tissues. There was no significant difference in DNA concentration between FF tissues and paired FFPE tissues. Moreover, the results of gene mutation analysis in our study demonstrated the concentration of DNA extracted from needle 


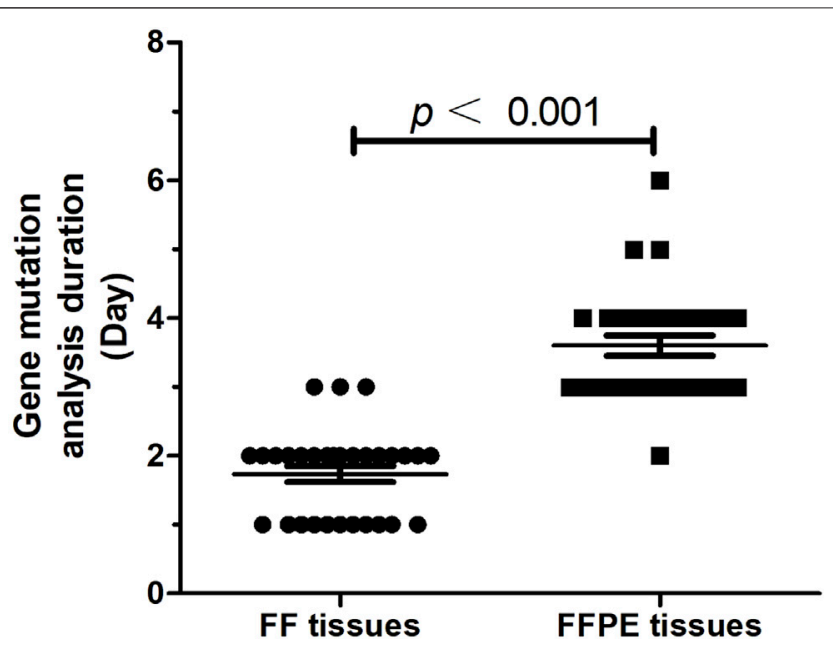

FIGURE 5 | The time interval (day) between physicians requesting and obtaining a test result $(n=30)$.

biopsy samples was adequate for multi-gene mutation testing. Indeed, Cheung et al. [15] reported that CT-guided core-needle biopsy of advanced NSCLC enables the acquisition of sufficient tissue for EGFR mutational analysis. In addition, no significant difference in $\mathrm{Ct}$ value was observed in ARMS-PCR systems between FF tissues and paired FFPE tissues. The $\mathrm{Ct}$ value of a reaction is defined as the cycle number when the fluorescence of a PCR product can be detected above the background signal. Therefore, the result of the study means the number of DNA templates was equal between FF tissues and paired FFPE tissues, though formaldehyde-driven cross-linking between proteins and nucleic acids.

In our study, $61.0 \%$ of patients carried EGFR mutations among 77 patients. According to a worldwide prospective study, Asian patients with lung adenocarcinoma showed 51.4\% EGFR overall mutation frequency [16]. In the mainland of China, the overall EGFR mutation rate is $50.2 \%$ [17]. Our present study showed the overall mutation rate of EGFR was $61.0 \%$, which is higher than the levels in the Asian and China mainland. Moreover, FF tissues in two cases revealed two gene mutations, while just one gene mutation was detected in the paired FFPE tissues. FF tissues from one case of SCC showed PIK3CA mutation, while the paired FFPE tissues showed wild-type status. However, histological heterogeneity was not found in FF tissues and paired FFPE tissues from the three cases. These results suggest gene testing using fresh tissues might increase gene mutations detection rates. We speculate that the fresh tissues from NSCLC seem to preserve more variants data than FFPE tissues. Indeed, Verhoest et al. [18] found only half of the true VHL abnormalities were identified in FFPE tissues as compared with that in FF tissues. However, Case 19 in our study showed wild-type status in FF tissues with pure acinar histology, while the paired FFPE tissues showed EGFR 20-ins with a predominant micropapillary pattern. This inconsistency can be attributed to the intratumor histological and genetic heterogeneity [19]. Micropapillary adenocarcinoma is considered equivalent to poorly differentiated adenocarcinoma and resembles morphology associated with high-grade lesions.
Moreover, the EGFR-mutated biopsy samples from the advanced lung adenocarcinoma had a higher frequency of micropapillary pattern than EGFR-wild type tumors [20]. In spite of the potential problems of both sampling variation and tumor heterogeneity, our study shows that multi-gene mutation analysis using FF samples may provide valid, robust data.

Although the fresh tissue has distinct advantages in gene analysis, the main limitation is that in fresh material, it is impossible to accurately evaluate the histological classification of tumor and estimate the tumor cell density, and this can lead to false-negative results. In our study, the frozen section examination is a rapid and reliable method for tumor diagnosis. It can accurately distinguish NSCLC from small cell carcinoma, sclerosing pneumocytoma, granulomatous lung disease, etc. Besides, the frozen section examination will also provide the data of tumor cell count to guarantee the success rate of gene analysis. Accordingly, we develop a procedure of gene mutation testing using fresh core needle biopsy specimens of NSCLC. As shown in Figure 6, samples from patients with solid pulmonary masses or suspected lung cancer were obtained by percutaneous CT-guided core needle biopsy. Frozen section examination using fresh biopsy samples quickly classified the masses as convinced NSCLC, diseases except for NSCLC, and uncertain diagnosis. The fresh samples diagnosed with convinced NSCLC were used to extract DNA and subsequent multi-gene mutation analysis. If NSCLC was not confirmed by frozen section examination, the fresh samples were fixed in $10 \%$ formalin and prepared for paraffin sections. Immunohistochemistry (IHC) was carried out when necessary. For example, an IHC panel that includes thyroid transcription factor-1 (TTF-1), Napsin A, CK5/ 6, p40, CD56, synaptophysin (Syn), and chromogranin (CgA) will be useful in the differentiation of the histological type of lung cancers [21]. The procedure will prevent misuse of molecular testing in lung cancer. If NSCLC was confirmed by final pathology diagnosis, FFPE tissues were used to extract DNA and multi-gene mutation analysis. The clinical treatment strategy for patients was optimized based on gene test results. Using this procedure of gene mutation testing, the time interval between physicians requesting and obtaining a test result has been shortened to fewer than 2 days. In Asian, the mean time interval for reporting the test was 17.6 days [17]. After all, timely treatment can delay progression and prolong the survival of patients with advanced NSCLC [22].

There are limitations to our study. First, there is a considerable risk of misdiagnosis during frozen section examination. We think pathologist experience is of crucial importance for the diagnostic accuracy of frozen sections. Moreover, the uncertain diagnosis of fresh tissues will not be allowed into the procedure of gene testing. In addition, careful clinical evaluation combined with pathology diagnosis may reduce the misdiagnosis of lung nodules. Second, in our study, the sensitivity and specificity of gene testing using FF tissues are 96 and 75\% when compared with FFPE tissues. The high sensitivity and low specificity may be attributed to the selection of cases through frozen section examination. More cases are necessary to evaluate the specificity and sensitivity in future studies. However, the high coincidence rate $(93.3 \%)$ of gene testing suggests a reliable detection procedure using FF tissues in our study.

In conclusion, we performed multi-gene mutation testing using fresh needle biopsy samples from patients with 


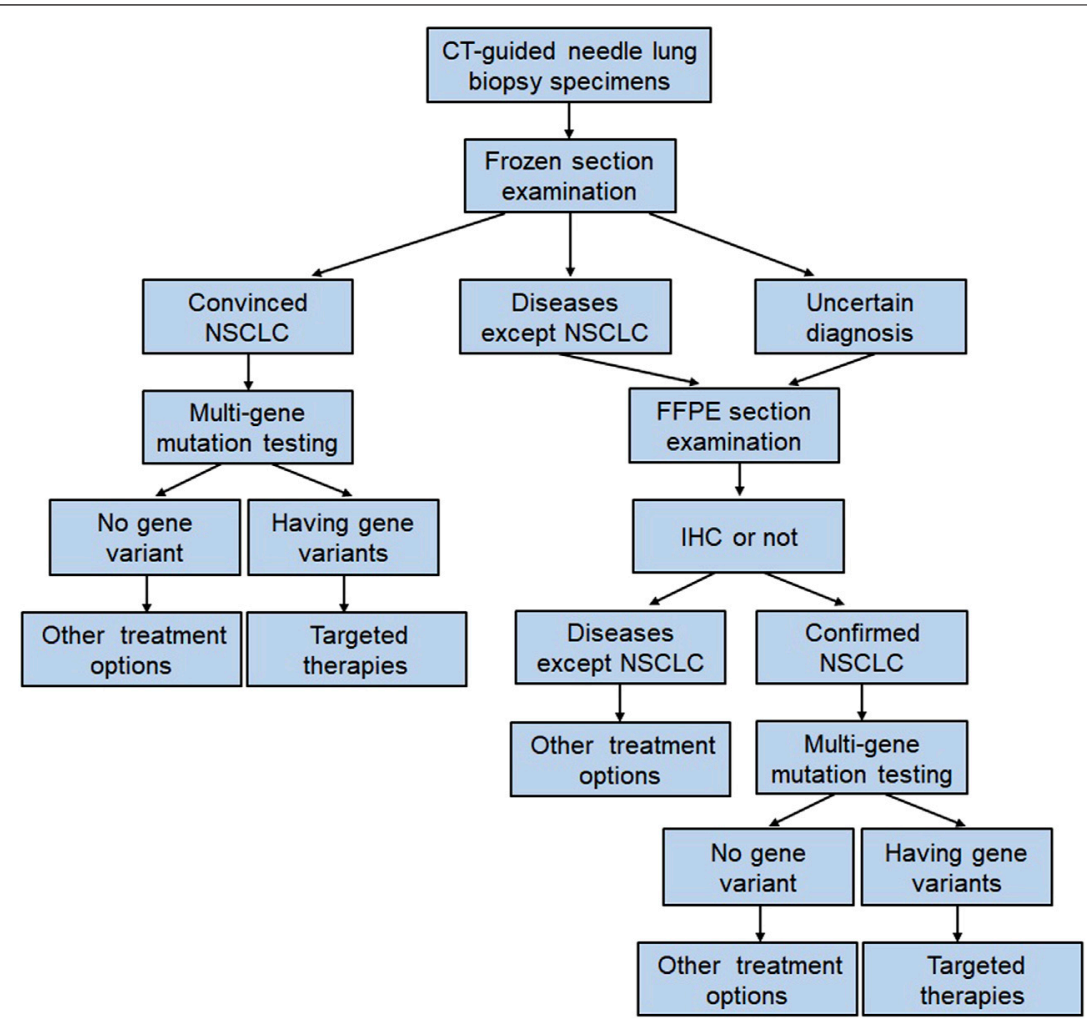

FIGURE 6 | The multi-gene mutation testing procedure using CT-guided core needle lung biopsy samples. Abbreviations: FFPE, formalin-fixed paraffin-embedded; $\mathrm{HC}$, immunohistochemistry.

NSCLC. Our testing results indicated that: 1) It is feasible to detect multi-gene mutation using fresh needle biopsy samples of NSCLC. 2) Frozen section examination plays a critical role in the sample quality control, including pathological diagnosis of lung tumor and estimation of tumor cell count. 3) Fresh samples are more likely to increase the detection rate of gene mutations. 4) It greatly reduces the waiting time of patients, improves the efficiency of gene detection. In conclusion, our study demonstrates that multi-gene mutation testing using fresh core needle biopsy samples is a reasonable, achievable and quick approach. Fresh tissues from core needle biopsy serve as the potential alternative to FFPE tissues for multi-gene testing in patients with NSCLC.

\section{DATA AVAILABILITY STATEMENT}

The original contributions presented in the study are included in the article/supplementary material, further inquiries can be directed to the corresponding authors.

\section{ETHICS STATEMENT}

The studies involving human participants were reviewed and approved by Medical Ethical Committee of Shandong Provincial
Hospital. The patients/participants provided their written informed consent to participate in this study.

\section{AUTHOR CONTRIBUTIONS}

Study conception and design: XYe, XYa, XCS, and ZGY. Core needle biopsy for patients with NSCLC: ZGW, GHH, MM, XYH, JW, WHL, YN, XYa, and XYe. Pathology examination: ZGY, XKC, YYZ, JML, JWX, HYJ, and XCS. Gene testing: ZXC. Data collection and statistical analysis: ZGY. Writing of manuscript: ZGY. All authors read and approved the final manuscript.

\section{FUNDING}

This study was sponsored by the National Natural Science Foundation of China (81502610 and 82072028), Shandong Provincial Natural Science Foundation (ZR201911040313 and ZR2021MH095), Shandong Medical and Health Science Technology Development Program (2019WS488), Jinan Science and Technology Planning Project (202019032).

\section{CONFLICT OF INTEREST}

The authors declare that the research was conducted in the absence of any commercial or financial relationships that could be construed as a potential conflict of interest. 


\section{REFERENCES}

1. Valentino F, Borra G, Allione P, Rossi L. Emerging Targets in Advanced Non-SmallCell Lung Cancer. Future Oncol (2018) 14(13s):61-72. doi:10.2217/fon-2018-0099

2. Chen C-M, Chang JW-C, Cheung Y-C, Lin G, Hsieh J-J, Hsu T, et al. Computed Tomography-Guided Core-Needle Biopsy Specimens Demonstrate Epidermal Growth Factor Receptor Mutations in Patients with Non-Small-Cell Lung Cancer. Acta Radiol (2008) 49(9):991-4. doi:10.1080/02841850802294410

3. Lai Y, Zhang Z, Li J, Sun D, Zhou YA, Jiang T, et al. EGFR Mutations in Surgically Resected Fresh Specimens from 697 Consecutive Chinese Patients with Non-Small Cell Lung Cancer and Their Relationships with Clinical Features. Int J Mol Sci (2013) 14(12):24549-59. doi:10.3390/ijms141224549

4. Ye X, Wang J, Ni Y, Yang X, Huang G, Wei Z, et al. Diagnostic Ability of Percutaneous Core Biopsy Immediately after Microwave Ablation for Lung Ground-Glass Opacity. J Can Res Ther (2019) 15(4):755-9. doi:10.4103/ jcrt.JCRT_399_19

5. Wu S, Shi X, Si X, Liu Y, Lu T, Zhang L, et al. EGFR T790M Detection in Formalin-Fixed Paraffin-Embedded Tissues of Patients with Lung Cancer Using RNA-Based In Situ Hybridization: A Preliminary Feasibility Study. Thorac Cancer (2019) 10(10):1936-44. doi:10.1111/1759-7714.13169

6. Sung H, Ferlay J, Siegel RL, Laversanne M, Soerjomataram I, Jemal A, et al. Global Cancer Statistics 2020: GLOBOCAN Estimates of Incidence and Mortality Worldwide for 36 Cancers in 185 Countries. CA A Cancer J Clin (2021) 71(3):209-49. doi:10.3322/caac.21660

7. Chen W, Zheng R, Baade PD, Zhang S, Zeng H, Bray F, et al. Cancer Statistics in China, 2015. CA: A Cancer J Clinicians (2016) 66(2):115-32. doi:10.3322/caac.21338

8. Zhou C. Lung Cancer Molecular Epidemiology in China: Recent Trends. Transl Lung Cancer Res (2014) 3(5):270-9. doi:10.3978/j.issn.2218-6751.2014.09.01

9. Wu F, Wang L, Zhou C. Lung Cancer in China: Current and Prospect. Curr Opin Oncol (2021) 33(1):40-6. doi:10.1097/CCO.0000000000000703

10. Chinese Association of Oncologists. Chinese Society for Clinical Cancer Chemotherapy, Chinese Association of Oncologists Chinese Society for Clinical Cancer Chemotherapy. The Guideline for Diagnosis and Treatment of Chinese Patients with Sensitizing EGFR Mutation or ALK Fusion Gene-Positive Non-small Cell Lung Cancer (2015 Version). Zhonghua Zhong Liu Za Zhi (2015) 37(10):796-9. doi:10.3760/ cma.j.issn.0253-3766.2015.10.018

11. Campos PF, Gilbert TMP. DNA Extraction from Formalin-Fixed Material. Methods Mol Biol (2012) 840:81-5. doi:10.1007/978-1-61779-516-9_11

12. Ulivi P, Romagnoli M, Chiadini E, Casoni GL, Capelli L, Gurioli C. Assessment of EGFR and K-Ras Mutations in Fixed and Fresh Specimens from Transesophageal Ultrasound-Guided Fine Needle Aspiration in Non-Small Cell Lung Cancer Patients. Int J Oncol (2012) 41(1):147-52. doi:10.3892/ ijo.2012.1432

13. Suciu BA, Pap Z, Dénes L, Brînzaniuc K, Copotoiu C, Pávai Z. Allele-Specific PCR Method for Identification of EGFR Mutations in Non-Small Cell Lung Cancer: Formalin-Fixed Paraffin-Embedded Tissue Versus Fresh Tissue. Rom J Morphol Embryol (2016) 57(2):495-500.
14. Spencer DH, Sehn JK, Abel HJ, Watson MA, Pfeifer JD, Duncavage EJ. Comparison of Clinical Targeted Next-Generation Sequence Data from Formalin-Fixed and Fresh-Frozen Tissue Specimens. J Mol Diagn (2013) 15(5):623-33. doi:10.1016/j.jmoldx.2013.05.004

15. Cheung Y-C, Chang JW-C, Hsieh J-J, Lin G, Tsai Y-H. Adequacy and Complications of Computed Tomography-Guided Core Needle Biopsy on Non-Small Cell Lung Cancers for Epidermal Growth Factor Receptor Mutations Demonstration: 18-Gauge or 20-Gauge Biopsy Needle. Lung Cancer (2010) 67(2):166-9. doi:10.1016/j.lungcan.2009.04.007

16. Shi Y, Au JS-K, Thongprasert S, Srinivasan S, Tsai C-M, Khoa MT, et al. A Prospective, Molecular Epidemiology Study of EGFR Mutations in Asian Patients with Advanced Non-Small-Cell Lung Cancer of Adenocarcinoma Histology (PIONEER). J Thorac Oncol (2014) 9(2):154-62. doi:10.1097/ JTO.0000000000000033

17. Shi Y, Li J, Zhang S, Wang M, Yang S, Li N.,et al. Molecular Epidemiology of EGFR Mutations in Asian Patients with Advanced Non-Small-Cell Lung Cancer of Adenocarcinoma Histology - Mainland China Subset Analysis of the PIONEER Study. PLoS One (2015) 10(11):e0143515. doi:10.1371/ journal.pone. 0143515

18. Verhoest G, Patard J-J, Fergelot P, Jouan F, Zerrouki S, Dreano S, et al. Paraffin-Embedded Tissue Is Less Accurate Than Frozen Section Analysis for Determining VHL Mutational Status in Sporadic Renal Cell Carcinoma. Urol Oncol Semin Original Invest (2012) 30(4):469-75. doi:10.1016/ j.urolonc.2010.07.005

19. Marino FZ, Bianco R, Accardo M, Ronchi A, Cozzolino I, Morgillo F, et al. Molecular Heterogeneity in Lung Cancer: From Mechanisms of Origin to Clinical Implications. Int J Med Sci (2019) 16(7):981-9. doi:10.7150/ ijms.34739

20. Matsumura M, Okudela K, Kojima Y, Umeda S, Tateishi Y, Sekine A, et al. A Histopathological Feature of EGFR-Mutated Lung Adenocarcinomas with Highly Malignant Potential - An Implication of Micropapillary Element. Plos One (2016) 11(11):e0166795. doi:10.1371/ journal.pone. 0166795

21. Zhang C, Schmidt LA, Hatanaka K, Thomas D, Lagstein A, Myers JL. Evaluation of Napsin A, TTF-1, P63, P40, and CK5/6 Immunohistochemical Stains in Pulmonary Neuroendocrine Tumors. Am J Clin Pathol (2014) 142:320-4. doi:10.1309/AJCPGA0IUA8BHQEZ

22. Sheinson D, Wong WB, Wu N, Mansfield AS. Impact of Delaying Initiation of Anaplastic Lymphoma Kinase Inhibitor Treatment on Survival in Patients with Advanced Non-Small-Cell Lung Cancer. Lung Cancer (2020) 143:86-92. doi:10.1016/j.lungcan.2020.03.005

Copyright () 2021 Yao, Wei, Cheng, Huang, Zong, Meng, Li, Han, Xu, Wang, Jing, $\mathrm{Li}$, Cao, Ni, Sun, Yang and Ye. This is an open-access article distributed under the terms of the Creative Commons Attribution License (CC BY). The use, distribution or reproduction in other forums is permitted, provided the original author(s) and the copyright owner(s) are credited and that the original publication in this journal is cited, in accordance with accepted academic practice. No use, distribution or reproduction is permitted which does not comply with these terms. 